\title{
Proposal of Simple and Accurate Two-Parametric Approximation for the Q-Function
}

\author{
Jelena Nikolić, Zoran Perić, and Aleksandar Marković \\ Faculty of Electronic Engineering, University of Niš Department of Telecommunications, Aleksandra Medvedeva 14, 18000 Niš, Serbia \\ Correspondence should be addressed to Jelena Nikolić; jelena.nikolic@elfak.ni.ac.rs
}

Received 20 July 2017; Revised 10 October 2017; Accepted 8 November 2017; Published 31 December 2017

Academic Editor: Hua Fan

Copyright (c) 2017 Jelena Nikolić et al. This is an open access article distributed under the Creative Commons Attribution License, which permits unrestricted use, distribution, and reproduction in any medium, provided the original work is properly cited.

\begin{abstract}
The approximations for the Q-function reported in the literature so far have mainly been developed to overcome not only the difficulties, but also the limitations, caused in different research areas, by the nonexistence of the closed form expression for the $Q$-function. Unlike the previous papers, we propose the novel approximation for the Q-function not for solving some particular problem. Instead, we analyze this problem in one general manner and we provide one general solution, which has wide applicability. Specifically, in this paper, we set two goals, which are somewhat contrary to each other. The one is the simplicity of the analytical form of $Q$-function approximation and the other is the relatively high accuracy of the approximation for a wide range of arguments. Since we propose a two-parametric approximation for the $Q$-function, by examining the effect of the parameters choice on the accuracy of the approximation, we manage to determine the most suitable parameters of approximation and to achieve these goals simultaneously. The simplicity of the analytical form of our approximation along with its relatively high accuracy, which is comparable to or even better than that of the previously proposed approximations of similar analytical form complexity, indicates its wide applicability.
\end{abstract}

\section{Introduction}

It is well known that, due to the prevalence of Gaussian random variables, the one-dimensional Gaussian $Q$-function, or shortly Q-function, represented as the complement of the cumulative distribution function of the Gaussian random variable $X$ with zero mean and unit variance

$$
Q(x)=\frac{1}{\sqrt{2 \pi}} \int_{x}^{\infty} \exp \left\{-\frac{t^{2}}{2}\right\} d t
$$

is one of the most frequently encountered nonelementary integrals in the field of applied mathematics and statistics [120]. The impossibility of expressing the $Q$-function in a closed form in terms of elementary functions made many of the analyses carried out in the field of signal processing and communication theory complex, or even limited. The solution of the observed problem was somewhat found in approximating the $Q$-function. As shown in [1-20] the approximations for the $Q$-function not only facilitate performance analyses of various communication systems, but also provide further mathematical analyses limited by the nonexistence of the closed-form formula for the Q-function. Recall here that a Gaussian probability density function (PDF) characterizes speech signals, signals in wireless receivers, and OFDM modulated signals $[15,16,21-28]$, so that the suitable solution to the problem of the $Q$-function approximation we observe in this paper is of significance in many application areas. For instance, as shown in $[2,3,10-12]$, the problem of the Qfunction approximation is of importance in the evaluation of the symbol error probability (SEP) of digital modulations in the presence of additive white Gaussian noise and the average SEP over fading channels. As shown in [9], this problem also occurs in the field of dynamic spectrum access in cognitive radio networks. Moreover, this problem is present in the field of quantization of the Gaussian source [19]. In particular, with the goal to derive closed form formulas enabling a straightforward performance analysis of the quantizers designed for the Gaussian source, some complex forms of the $Q$-function approximation have recently been applied in $[21,22]$. Also, in a number of papers, it has been pointed out that certain difficulties appear due to the nonexisting closedform solution for the inverse Q-function and other related 
special functions, for instance, in [29-33]. This has motivated the research presented in $[29,30,33]$, where piecewise linear solutions have been proposed to overcome somewhat this problem, where, as a consequence, the performance degradation has been noticed. As indicated in $[19,28]$, with the suitable approximation of the $Q$-function this problem can be better solved, so that this has directed the research we presented here. Eventually, one should highlight here that the solution to the problem of the Q-function approximation can also be helpful in many analyses involving the error function, gamma function, and incomplete gamma function, which are directly related to the $Q$-function.

A notable amount of empirical and analytical approximation methods have been reported in the literature, for instance, in [1-19], providing different trade-offs between the accuracy of the $Q$-function approximation and its analytical tractability. Specifically, many of the available $Q$-function approximations have very complex analytical forms, which are not tractable [13]. Also, as shown in [19], some of the available $Q$-function approximations, in spite of having suitable analytical tractability, still provide an insufficient accuracy. Eventually, some of the methods are suitable for small arguments and not for large arguments and vice versa. As highlighted in the survey on approximations of the Qfunction reported in [13], due to these drawbacks of the existing Q-function approximations this problem is still present and widespread. This has inspired the research presented in this paper where the goal is to propose one very accurate novel approximation of the $Q$-function having relatively simple analytical form. In other words, in this paper we focus toward two goals, one is a high accuracy of the Q-function approximation for any argument of the approximation and the second is the simplicity of the approximation analytical form, which provides performing of many analyses involving the $Q$-function in a simple manner.

A brief summary of the remainder of the paper is as follows. Section 2 provides an overview of the previous work related to the $Q$-function approximations. Section 3 proposes the novel two-parametric $Q$-function approximation and describes the methodology for determining the particular parameter values. Section 4 discusses the obtained numerical results, where the focus is on analyzing the effect of the choice of the particular parameter values on the relative error of approximation in order to determine the most suitable form of our approximation in terms of accuracy and to enable its further application in any Q-function approximation problems. Section 4 also provides the comparison of the novel approximation in terms of accuracy with the other existing $Q$ function approximations from the literature having similar analytical form complexity. Section 5 summarizes the key features of the novel $Q$-function approximation and points out the contributions achieved in the paper.

\section{Previous Work on the Approximation of the Q-Function}

This section provides a brief overview of the previous work related to the $Q$-function approximations. In particular, the Q-function approximations, having both relatively simple analytical forms and relatively high accuracies for a specific range of argument values, are mainly presented here with the goal to provide a fair comparison with the $Q$-function approximation we propose in this paper. Let us denote with $F^{[\text {Karagiannidis and Lioumpas] }}(x)$ the $Q$-function approximation given in [2, Eq. (6)] by Karagiannidis and Lioumpas.

$$
\begin{aligned}
& F^{[\text {Karagiannidis and Lioumpas }]}(x) \\
& =\frac{(1-\exp \{-A x / \sqrt{2}\}) \exp \left\{-x^{2} / 2\right\}}{\sqrt{2 \pi} B x}, \\
& A=1.98, B=1.135,
\end{aligned}
$$

which have proved very accurate for small arguments. The values of the parameters $A$ and $B$ have been determined so that the integral of the absolute error for a wide range of arguments $(x \in[0,20])$ has been minimized [2]. As noted in [13], the Q-function approximation proposed in [3, Eq. (2)] by Jang

$$
\begin{aligned}
& F^{[\text {Jang }]}(x)=\frac{(1-\exp \{-C x\}) \exp \left\{-x^{2} / 2\right\}}{\sqrt{2 \pi} x}, \\
& \qquad C=\sqrt{\frac{\pi}{2}},
\end{aligned}
$$

has been built upon the approximation from [2], by setting $A=\sqrt{\pi}$ and $B=1$. This approximation has two main advantages compared to the one from [2]. The first one refers to the fact that it is the upper bound approximation of the Q-function, while the approximation from [2] is neither an upper bound nor a lower bound approximation of the $Q$-function. Another advantage of $F^{[J a n g]}(x)$ over $F^{[\text {Karagiannidis and Lioumpas] }}(x)$ refers to the fact that unlike the approximation from [2], with the one from [3] the absolute relative error of approximation approaches zero in the tail, that is, for $x \rightarrow \infty$.

Further simplification of the approximations from $[2,3]$ has been achieved in [4, Eq. (18)] by Fan, where Mill's ratiobased form upper bound expression for the $Q$-function has been proposed

$$
F^{[\mathrm{Fan}]}(x)=\frac{1}{\sqrt{2 \pi}} \frac{1}{\sqrt{x^{2}+1}} \exp \left\{-\frac{x^{2}}{2}\right\} .
$$

The comparison of the upper bound expressions from $[3,4]$ has shown that the one from [4] is a tighter approximation of the Q-function. Let us also review here the following Qfunction approximation from [5, Eq. (14)]:

$$
F^{[\text {Chiani et al. }]}(x)=\frac{1}{12} \exp \left\{-\frac{x^{2}}{2}\right\}+\frac{1}{4} \exp \left\{-\frac{2 x^{2}}{3}\right\},
$$

given by Chiani et al., which has the sum of exponential function based form. This approximation has been reported in a number of papers on the subject above $[2,3,8-$ 13, 17-19] due to its very simple analytical form. Comparing the Q-function approximations $F^{[\text {Chiani et al.] }}(x)$ and 
$F^{[\text {Karagiannidis and Lioumpas }]}(x)$ it has been concluded in [2] that $F^{\text {[Karagiannidis and Lioumpas] }}(x)$ has a little more complex form but provides a tighter approximation for a wide range of arguments, especially in the small arguments region.

In this paper, for the comparison purposes we also review the latest reported Q-function approximation from [19, Eq. (14)] given by Nikolić et al.

$$
\begin{aligned}
& F^{[\text {Nikolić et al. }]}(x)=\frac{1}{\sqrt{2 \pi}} \frac{1}{\sqrt{x^{2}+a}} \exp \left\{-\frac{x^{2}}{2}\right\} \\
& \\
& 1 \leq a<2,
\end{aligned}
$$

which has proven an upper bound approximation for $x>$ $\sqrt{\left(\sqrt{4 a+1}+2 a^{2}-2 a-1\right) /(4-2 a)}$. The particular forms of this approximation, that is, the particular values of the parameter $a$, have been determined in [19] in order to most successively solve the specific problem arising in variancematched scalar quantization of Gaussian source of unit variance, which is caused by the nonexistence of the closed form formula for the $Q$-function. In other words, unlike this paper, where the problem of determining the particular form of the parametric Q-function approximation is posed and solved in one general manner, in [19], the forms of the Qfunction approximations have been determined for the given set of the Q-function arguments, which are of interest for performance analysis of Gaussian source scalar quantizers. In order to provide a fair comparison with the results from [19] in the numerical results section we compare the accuracies of our novel approximation with the ones of the approximations from [19, Eq. (38), Eq. (39), Eq. (44)], where the parameter $a$ amounts to $a=1.579, a=1.784$, and $a=0.1178 x+1.3742$, respectively.

As we have already highlighted, there is always a trade-off between the accuracy and simplicity of the analytical form of approximation. This trade-off is apparent in the upper and lower bound approximations of the Q-function proposed in [8, Eqs. (12) and (13)] by Abreu

$$
\begin{aligned}
F^{[\text {Abreu }] \text { upp }}(x)= & \frac{1}{50} \exp \left\{-x^{2}\right\} \\
& +\frac{1}{2(x+1)} \exp \left\{-\frac{x^{2}}{2}\right\}, \\
F^{[\text {Abreu }] \text { low }}(x)= & \frac{1}{12} \exp \left\{-x^{2}\right\} \\
& +\frac{1}{\sqrt{2 \pi}} \frac{1}{(x+1)} \exp \left\{-\frac{x^{2}}{2}\right\} .
\end{aligned}
$$

Namely, the last two approximations of the Q-function have more complex forms compared to the approximations given by (2)-(6), justified by the somewhat improved approximation accuracy. Recall here that, as it has been highlighted in the number of papers on the subject above, the more complex forms of the $Q$-function approximations have been developed bearing in mind applications that require high estimation accuracies. Let us conclude here that in spite of their relatively simple analytical form, all of the reviewed approximations do not provide high accuracy for any argument value we are intending to reach by the proposal of this paper.

\section{Main Result}

The main result of this paper is the novel Q-function approximation, which is described in detail in what follows. What we propose is the parametric approximation of the form:

$$
F^{p}(x)=\frac{1}{\sqrt{2 \pi}} \frac{1}{\sqrt{x^{2}+c_{1} x+c_{2}}} \exp \left\{-\frac{x^{2}}{2}\right\},
$$

which is defined by only two parameters denoted by $c_{1}$ and $c_{2}$. The values of the parameter $c_{2}$ are determined straightforwardly from the following conditions:

$$
\begin{aligned}
\lim _{x \rightarrow 0} F^{p}(x) & =\frac{1}{\sqrt{2 \pi}} \frac{1}{\sqrt{c_{2}}}, \\
Q(0) & =0.5, \\
\lim _{x \rightarrow 0} F^{p}(x) & =0.5 .
\end{aligned}
$$

As a result, we obtain

$$
c_{2}=\frac{2}{\pi}=0.6366
$$

that is, our approximation gets the form

$$
F^{p}(x)=\frac{1}{\sqrt{2 \pi}} \frac{1}{\sqrt{x^{2}+c_{1} x+0.6366}} \exp \left\{-\frac{x^{2}}{2}\right\} .
$$

Observe that the manner for determining the values of the other parameter, denoted by $c_{1}$, will be described as we proceed. Here it is important to highlight the following nice properties of our approximation, which can be mathematically formulated as

$$
\begin{aligned}
\lim _{x \rightarrow 0}\left(F^{p}(x)-Q(x)\right) & =0, \\
\lim _{x \rightarrow \infty}\left(\frac{F^{p}(x)}{Q(x)}\right) & =1, \\
\left|c_{1}\right| & <\sqrt{\frac{8}{\pi}} .
\end{aligned}
$$

Among the approximations reviewed in the previous section, the only one having these nice properties are the ones from [3], which due to involving the two exp functions in the Q-function approximation can be considered a slightly complex approximation compared to the proposed one.

In order to optimize our $Q$-function approximation in terms of its parameter $c_{1}$ with respect to absolute relative error (RE) of approximation

$$
\mathrm{RE}(x)=\frac{|F(x)-Q(x)|}{Q(x)},
$$


we define the two criterions and, as a result, we obtain the values of the parameters denoted by $c_{1}{ }^{\mathrm{I}}$ and $c_{1}{ }^{\mathrm{II}}$, respectively. With the first criterion we determine the parameter $c_{1}=c_{1}{ }^{\mathrm{I}}$ of our approximation so that the average RE in approximating the Q-function is minimized for the given range of argument values and an arbitrary number $M$ of numerical values $x_{m}$ employed for the argument $x$ [34]. Mathematically, this can be formulated as follows:

$$
c_{1}{ }^{\mathrm{I}}=\underset{c_{1}}{\arg \min }\left\{\frac{1}{M}\left[\sum_{m=1}^{M} \frac{\left|(1 / \sqrt{2 \pi})\left(1 / \sqrt{x_{m}{ }^{2}+c_{1} x_{m}+0.6366}\right) \exp \left\{-x_{m}{ }^{2} / 2\right\}-Q\left(x_{m}\right)\right|}{Q\left(x_{m}\right)}\right]\right\} .
$$

The result of this optimization is the particular value of the parameter $c_{1}{ }^{\mathrm{I}}$. With the second criterion, formally formulated as follows

$$
c_{1}{ }^{\mathrm{II}}=\underset{c_{1}}{\arg \min }\left\{\max _{m}\left\{\frac{\left|(1 / \sqrt{2 \pi})\left(1 / \sqrt{x_{m}{ }^{2}+c_{1} x_{m}+0.6366}\right) \exp \left\{-x_{m}{ }^{2} / 2\right\}-Q\left(x_{m}\right)\right|}{Q\left(x_{m}\right)}\right\}\right\},
$$

the values of the parameter $c_{1}{ }^{\mathrm{II}}$ are determined so as to minimize the maximum absolute relative error for the observed set of numerical values $x_{m}$ employed for the argument $x$. Let us highlight here that the first criterion has also been utilized in [19] with the goal to determine the particular value of the parameter in [19, Eq. (14)], here in Eq. (6), while the second one has not been utilized. In particular, in [19] this criterion has been applied to the finite small set of the Qfunction arguments being of interest for solving the particular problem arising in Gaussian source scalar quantization, while in this paper, for the novel form of the $Q$-function, we assume the common region of the $Q$-function arguments and we apply these two criteria to exactly this region. Let us also highlight here that by specifying the above mentioned criteria in general forms, we have infect provided the manner for their application to any argument range, which makes our proposal more applicable.

\section{Discussion with Numerical Results Analysis}

In this section we compare the accuracy of the Q-function approximation proposed in the previous section with the ones of the other existing $Q$-function approximations from the literature, reviewed in Section 2, having similar analytical form complexity. In other words, in this section we demonstrate the validity of the proposed $Q$-function approximation by comparing its features with the ones of the subset of the previously proposed $Q$-function approximations. This subset of approximations has been carefully chosen to provide a fair comparison. Note that the accuracy is here viewed through the absolute relative error (RE) of approximating the $Q$ function, where it holds the higher RE, the smaller accuracy, and vice versa. First, for the proposed approximation and for the limited finite small set of the $c_{1}$ parameter numerical values $\left(c_{1} \in C=\{0.1,0.2,0.3,0.4,0.5,0.6,0.7,0.8,0.9,1.0\}\right)$ we study the effect of this parameter choice on the approximation accuracy. Based on this study, for the given set of the $c_{1}$ parameter values, we derive the conclusion about the most suitable value of the parameter in question. Since we obtain the solution of the formulated problem constrained to $C$ in such a manner, we also solve the problem in an unconstrained manner obtaining the most suitable particular forms of the novel approximation for the two specified criteria. To the end of this section we also provide one manner for further improving the accuracy of the proposed approximation at the cost of the slight increase of the analytical form complexity.

For the comparison purposes, we have calculated the absolute relative error of approximating the $Q$-function by using (14), where for $F(x)$ we have assumed the approximated functions from $[2-5,8,19]$ given by (2)-(7), and we have also assumed that $F(x)=F^{p}(x)$, whereas $F^{p}(x)$ is our proposal given by (11). The corresponding REs are denoted by $\mathrm{RE}^{[\text {Karagiannidis and Lioumpas }]}(x), \quad \mathrm{RE}^{[\mathrm{Jang}]}(x), \quad \mathrm{RE}^{[\mathrm{Fan}]}(x)$, $\mathrm{RE}^{[\text {Chiani et al.] }}(x), \quad \mathrm{RE}^{[\text {Nikolić et al.] }}(x), \quad \operatorname{RE}^{\text {upp[Abreu] }}(x)$, $\mathrm{RE}^{\text {low[Abreu }]}(x)$, and $\mathrm{RE}^{p}(x)$, respectively. Similarly as in the number of papers on the described subject for the comparison purposes we have chosen the common interval of argument $x$ ranging $[0,6]$. For such a specified argument region and for the approximations given by (2)-(5) and (7) we have determined the dependences of the absolute relative errors of approximating the Q-function on the argument $x$, presented in Figure 1. Along with these dependencies, in order to provide a more detailed comparison of the proposed approximation features to those of the approximations used for comparison purposes from [2-5, 8, 19], for the observed argument region, we have also determined the average values of RE and also of the maximum of RE (see Table 1). Figure 1 and Table 1 indicate that the upper bound approximation from [8] is generally the most accurate one (observe 


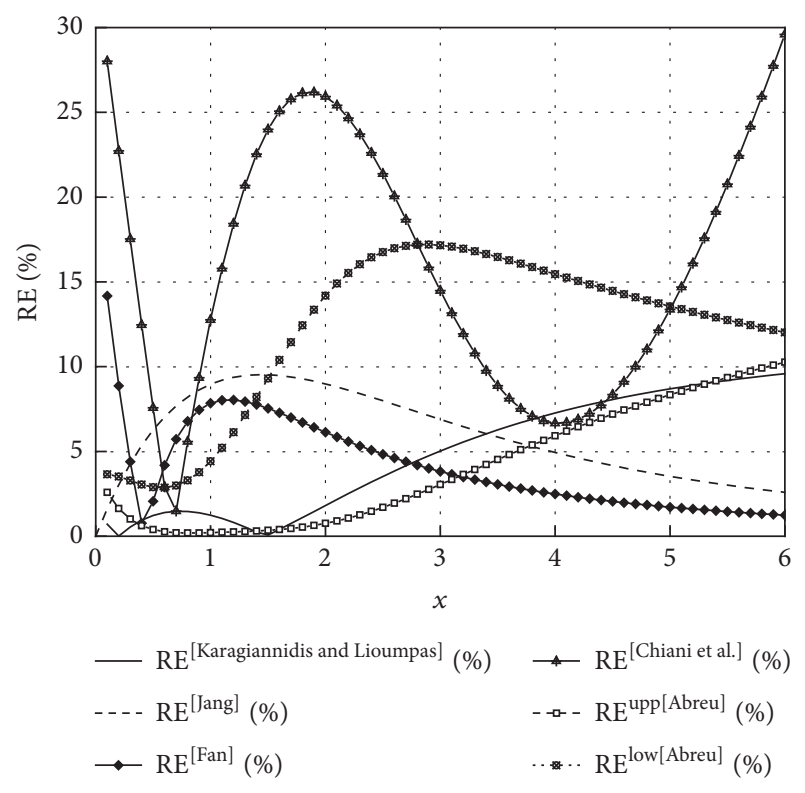

FIGURE 1: The comparison of REs for the subset of previously proposed approximations $[2-5,8]$.

$\left.\operatorname{RE}^{\text {upp }[\operatorname{Abreu}]}(x)\right)$. However, this approximation has more complex analytical form compared to the other presented approximations. In contrast, the approximation proposed by Chiani et al. [5] is less accurate, but it is an analytically very tractable and simple approximation. In a number of papers addressing the presented problem of the $Q$-function approximation (e.g., in $[2,10,11,19])$ it has been highlighted that the approximation $F^{\text {[Karagiannidis and Lioumpas] }}(x)$ performs well, especially for very small arguments, in terms of how accurately it resembles the $Q$-function combined with its relatively simple analytical form. In accordance with this observation, as well as from the dependencies presented in Figure 1, one can conclude that the approximation proposed by Karagiannidis and Lioumpas is the most accurate one for very small argument values. However, this approximation is less accurate as the argument value increases. As highlighted in $[3,13]$, the advantage of Jang's approximation [3] compared to the Karagiannidis and Lioumpas's approximation [2] is that the one from [3] is an upper bound approximation and that for very large arguments $(x \rightarrow \infty)$ Jang's approximation approaches zero, while the one proposed by Karagiannidis and Lioumpas approaches a constant. From the comparison of $\mathrm{RE}^{[\mathrm{Jang}]}(x)$ and $\mathrm{RE}^{[\mathrm{Fan}]}(x)$ one can conclude that $F^{[\mathrm{Fan}]}(x)$ is a more accurate approximation for a wide range of arguments observed. However, for a very narrow range of small argument values, Fan's approximation is inadequate for application due to relatively high inaccuracy. On the other side, for very small arguments, Jang's approximation has one nice property that our novel approximation also has (see (12)), which resolves the problem observed with Fan's approximation.

Figures 2 and 3 present the RE dependences on the argument $x$, which we have determined by applying (11) and (14). These dependences refer to the particular cases of the novel
TABLE 1: Average REs and maximum REs for the Q-function approximations from $[2-5,8,19]$.

\begin{tabular}{lcc}
\hline & Average RE & Maximum RE \\
\hline Ref. [2] Karagiannidis and & 4.8618 & 9.5874 \\
Lioumpas & 5.9325 & 9.5258 \\
Ref. [3] Jang & 4.1279 & 14.1663 \\
Ref. [4] Fan & 15.9433 & 29.5985 \\
Ref. [5] Chiani et al. & 4.0705 & 10.2645 \\
Ref. [8] upp Abreu & 12.1816 & 17.2027 \\
Ref. [8] low Abreu & 3.88473 & 36.50362 \\
Ref. [19] Nikolić et al. $a=1.579$ & 4.68581 & 40.26311 \\
Ref. [19] Nikolić et al. $a=1.784$ & 2.81822 & 31.93637 \\
Ref. [19] Nikolić et al. & & \\
$a=0.1178 x+1.3742$ &
\end{tabular}

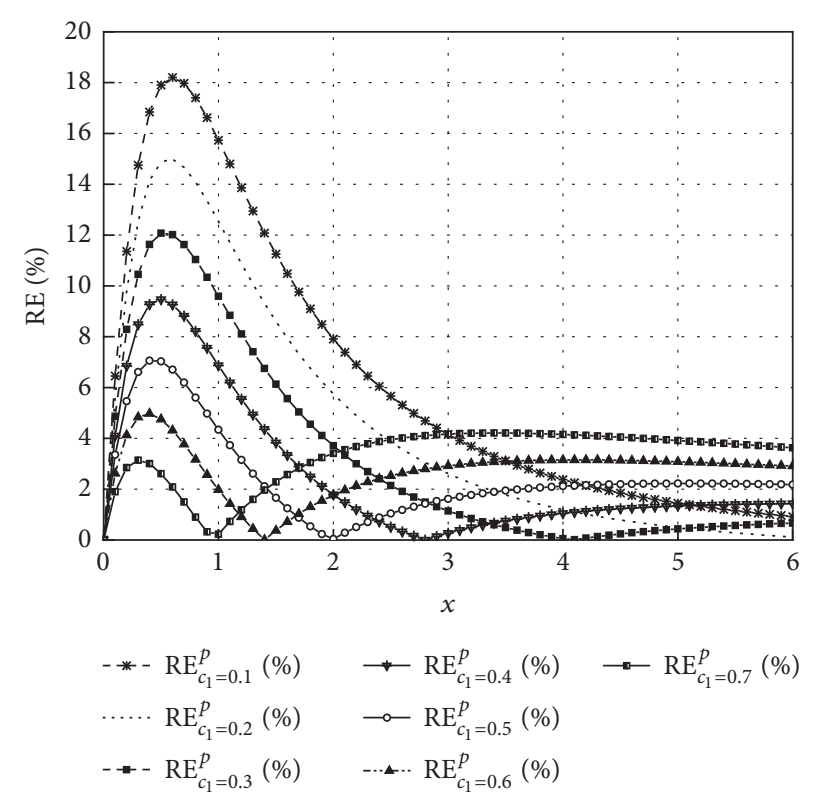

FIGURE 2: REs for our $Q$-function approximation, cases with $c_{1} \epsilon$ $\{0.1,0.2,0.3,0.4,0.5,0.6,0.7\}$.

parametric Q-function approximation, determined for the finite discrete set of $c_{1}$ parameter values $\left(c_{1} \in C=\{0.1,0.2\right.$, $0.3,0.4,0.5,0.6,0.7,0.8,0.9,1.0\})$. This finite small set of $c_{1}$ parameter values has been taken into the consideration with the goal to make our analysis simple and the results illustrative. Figures 2 and 3 and Table 2 demonstrate how important it is to study the effect of the choice of $c_{1}$ parameter value on the RE of the proposed approximation. Obviously, by increasing the values of $c_{1}$ parameter starting from $c_{1}=0.1$ to $c_{1}=0.5$ the average $\mathrm{RE}^{p}$ decreases and then with the further increase of $c_{1}$ values it began to increase. A similar conclusion can be derived by observing the maximum values of $\mathrm{RE}^{p}(x)$, where, for the given set of the $c_{1}$ parameter values $c_{1} \in$ $C=\{0.1,0.2,0.3,0.4,0.5,0.6,0.7,0.8,0.9,1.0\}$, the minimum of $\operatorname{RE}^{p}(x)$ maximums can be observed for $c_{1}=0.7$. Note that these are the solutions of the formulated problem constrained to $C$. By taking into account these findings, one can easily 


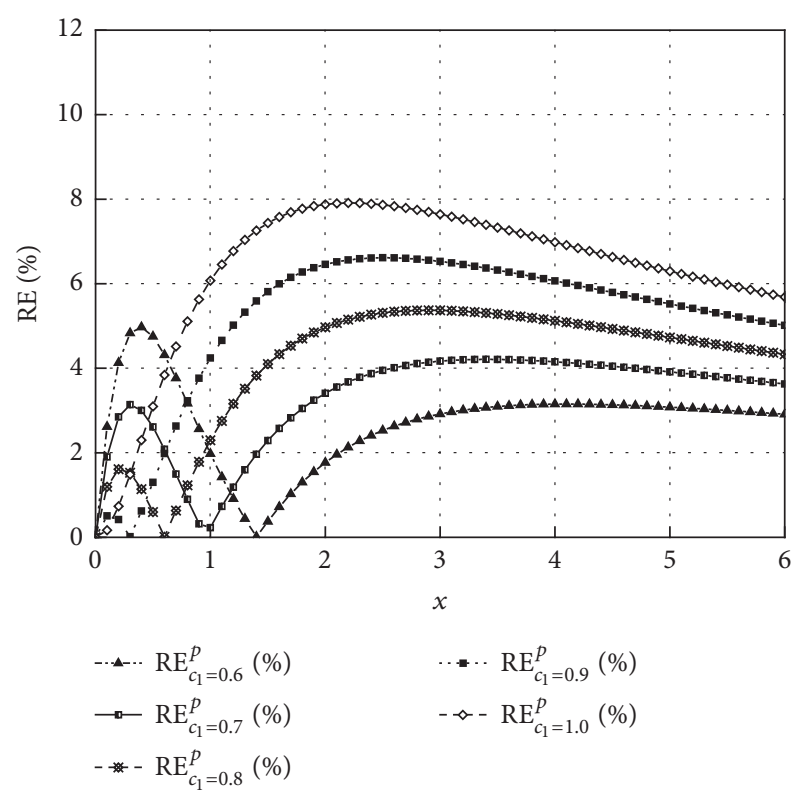

FIGURE 3: REs for our Q-function approximation, cases with $c_{1} \in$ $\{0.6,0.7,0.8,0.9,1.0\}$.

determine that the true minimum of the average $\mathrm{RE}^{p}$ and the minimum of $\mathrm{RE}^{p}(x)$ maximums are achieved at values of $c_{1}$ in the vicinity of $c_{1}=0.5$ and $c_{1}=0.7$, respectively. As a result, we obtain $c_{1}{ }^{\mathrm{I}}=0.48$ and $c_{1}{ }^{\mathrm{II}}=0.66$, which are the solution of the optimization problem specified by (15) and (16), for the region of the arguments we assumed. Accordingly, putting into (8) the particular values of the parameter $c_{1}$ determined by applying the two specified criterions, we obtain the following two proposals of our approximation:

$$
\begin{aligned}
& F^{p \mathrm{I}}(x)=\frac{1}{\sqrt{2 \pi}} \frac{1}{\sqrt{x^{2}+0.48 x+0.6366}} \exp \left\{-\frac{x^{2}}{2}\right\}, \\
& F^{p \mathrm{II}}(x)=\frac{1}{\sqrt{2 \pi}} \frac{1}{\sqrt{x^{2}+0.66 x+0.6366}} \exp \left\{-\frac{x^{2}}{2}\right\} .
\end{aligned}
$$

One can observe from Table 2 that by applying $F^{p I}(x)$ to the given range of argument values the average $\mathrm{RE}^{p}$ is indeed minimal and amounts to $2.4101 \%$. Also, one can observe from Table 2 that by applying $F^{p I I}(x)$ to the given range of argument values the maximum $\mathrm{RE}^{p}$ is indeed minimal and amounts to $3.8057 \%$. One interesting observation is that in the case with $c_{1}=c_{1}{ }^{\mathrm{II}}=0.66$ the average $\mathrm{RE}^{p}$ amounts to $3.0128 \%$ that is a slightly higher value than $2.4101 \%$, observed in the case with $c_{1}=c_{1}{ }^{\mathrm{I}}=0.48$. It is also interesting to notice from Tables 1 and 2 that, for the observed region of arguments, the proposed approximation provides a smaller average $\mathrm{RE}$ and also smaller maximum of RE compared to those of the previously proposed approximations reviewed here. This can be observed as an advantage of the proposed approximation compared to the listed ones. Moreover, by taking into account the convenient property of the proposed approximation stated by (13), one can conclude that, with the further extension of the range of values for the argument $x$,
TABLE 2: Average REs and maximum REs for the proposed Qfunction approximation and for a different values of $c_{1}$ parameter.

\begin{tabular}{lcc}
\hline & Average $\mathrm{RE}^{p}$ & ${\text { Maximum } \mathrm{RE}^{p}}$ \\
\hline$c_{1}=0.1$ & 6.2332 & 18.2024 \\
$c_{1}=0.2$ & 4.5439 & 14.9828 \\
$c_{1}=0.3$ & 3.2166 & 12.0742 \\
$c_{1}=0.4$ & 2.5583 & 9.4653 \\
$c_{1}=0.5$ & $\underline{2.4175}$ & 7.0567 \\
$c_{1}=0.6$ & 2.6871 & 4.9709 \\
$c_{1}=0.7$ & 3.2873 & $\underline{4.2099}$ \\
$c_{1}=0.8$ & 4.1371 & 5.3724 \\
$c_{1}=0.9$ & 5.1658 & 6.6146 \\
$c_{1}=1.0$ & 6.2837 & 7.9105 \\
$c_{1}=c_{1}{ }^{\mathrm{I}}=0.48$ & $\underline{\mathbf{2 . 4 1 0 1}}$ & 7.5045 \\
$c_{1}=c_{1}{ }^{\mathrm{I}}=0.66$ & 3.0128 & $\underline{\mathbf{3 . 8 0 5 7}}$ \\
$c_{1}=0.8, x<1 ; c_{1}=0.6, x \geq 1$ & $\mathbf{2 . 2 7 0 8}$ & $\mathbf{3 . 1 5 2 5}$ \\
\hline
\end{tabular}

we will again obtain $F^{\text {pII }}(x)$ as given by (18), which makes our proposal very useful and general.

From Figure 3 one can notice that, for the observed values of the parameter $c_{1}$, the accuracy of the novel approximation is noticeably good for very small arguments. Also, one can notice that starting from about $x \approx 1$ up to $x \approx 2$ the accuracy degrades as the argument value increases; that is, $\operatorname{RE}^{p}(x)$ increases with the argument value. Eventually, it is notable that the accuracy improves for $x>2$ with the further increase of the values of $x$. Although it cannot be seen from Figures 2 and 3 , by observing (13), one can anticipate that, with the further increase of $x, \operatorname{RE}^{p}(x)$ would gradually approach zero for all observed values of the parameter $c_{1}$. One interesting observation from Figure 3 is that, for $c_{1}=0.8$ and $x<1$, the proposed approximation is extremely accurate $\left(\mathrm{RE}^{p}<\right.$ $2 \%)$. From these figures one can also notice that, for $c_{1}=0.8$ and $x \geq 1$, the relative error of the proposed approximation increases at first and then gradually decreases. Moreover, one can derive an opposite conclusion when observing the proposed approximation for $c_{1}=0.6$ case. In other words, for the range of arguments $x<1$, the proposed approximation with the parameter $c_{1}$ equal to 0.6 is less accurate than in the case with $c_{1}$ equal to 0.8 , while for $x \geq 1$, the behaviour is reversed. For that reason, in what follows we also provide the corollary result of our analysis, that is, one composite solution to the observed problem, mathematically formulated as follows:

$$
\begin{aligned}
& F^{\text {com }}(x) \\
& =\left\{\begin{array}{l}
\frac{1}{\sqrt{2 \pi}} \frac{1}{\sqrt{x^{2}+0.8 x+0.6366}} \exp \left\{-\frac{x^{2}}{2}\right\}, x<1 \\
\frac{1}{\sqrt{2 \pi}} \frac{1}{\sqrt{x^{2}+0.6 x+0.6366}} \exp \left\{-\frac{x^{2}}{2}\right\}, x \geq 1,
\end{array}\right.
\end{aligned}
$$

which exploits the advantages of the novel approximations in the particular regions. The RE dependences on the argument $x$ determined for the cases where the approximated function are given by (17)-(19) are presented in Figures 4-10. For comparison purposes, the curves obtained by applying the approximation from $[2-4,8,19]$ have also been included 


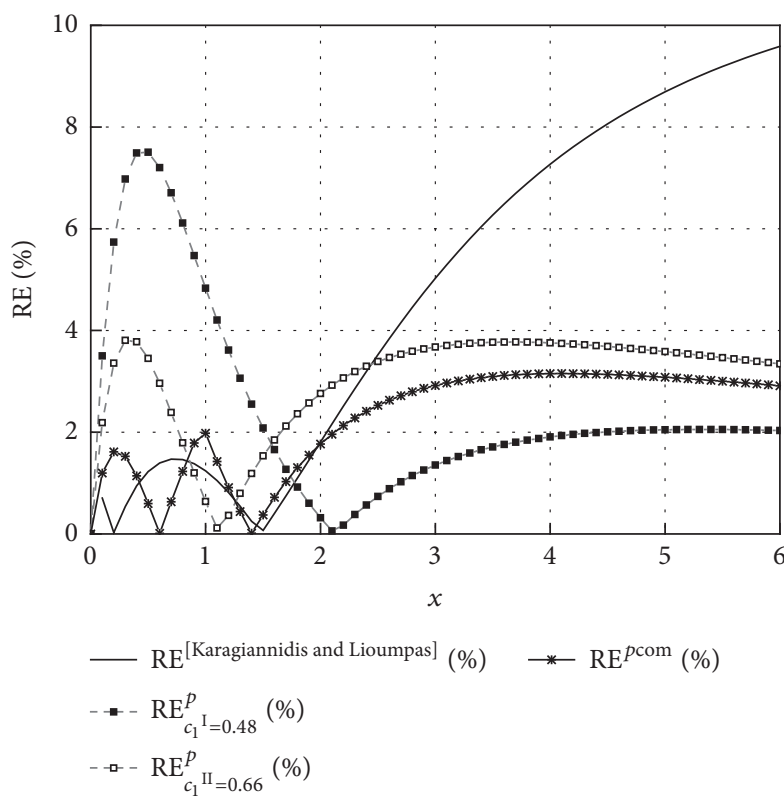

FIGURE 4: RE of the proposed approximation versus RE of the Karagiannids's et al. approximation.

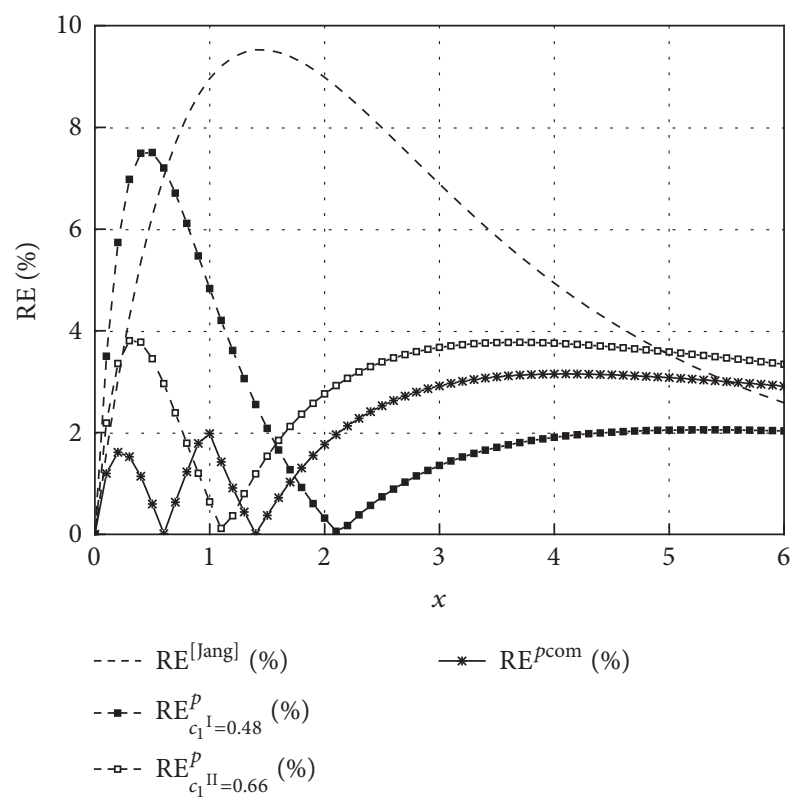

FIGURE 5: RE of the proposed approximation versus RE of Jang's approximation.

in these figures. Recall here that the results obtained by applying the approximation from [5] are very poor in terms of accuracy and, for that reason, have been omitted here. Obviously, with the approximation specified by (19) a notable improvement in terms of accuracy has been achieved. Specifically, as one can see from Table 2, with this composite solution we have managed to decrease both the average RE and the maximum of RE (see bolded values in Table 2), at the cost of the increase of analytical form complexity.

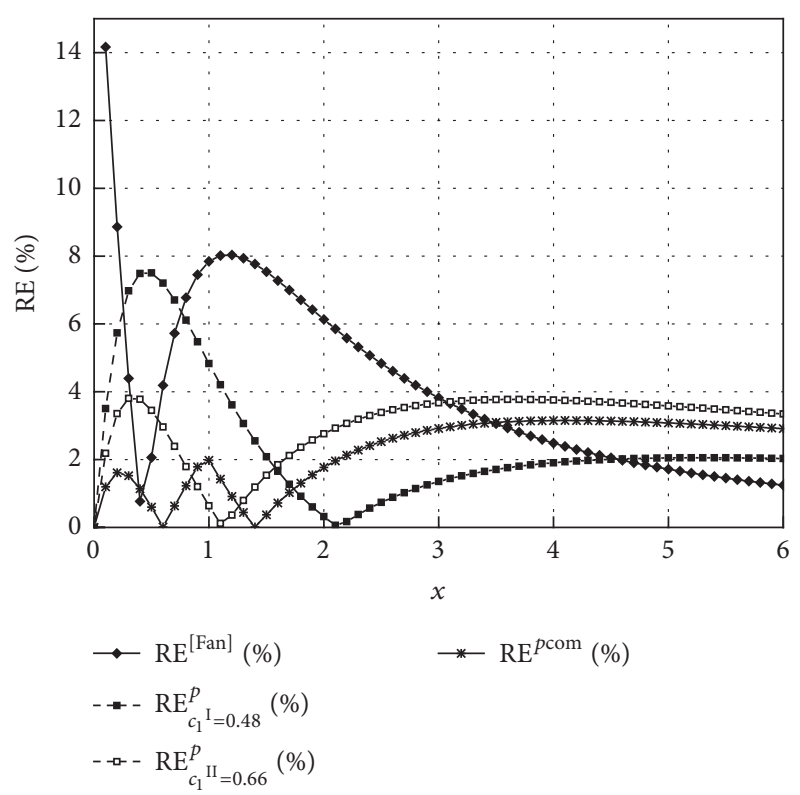

FIGURE 6: RE of the proposed approximation versus RE of the Fan's approximation.

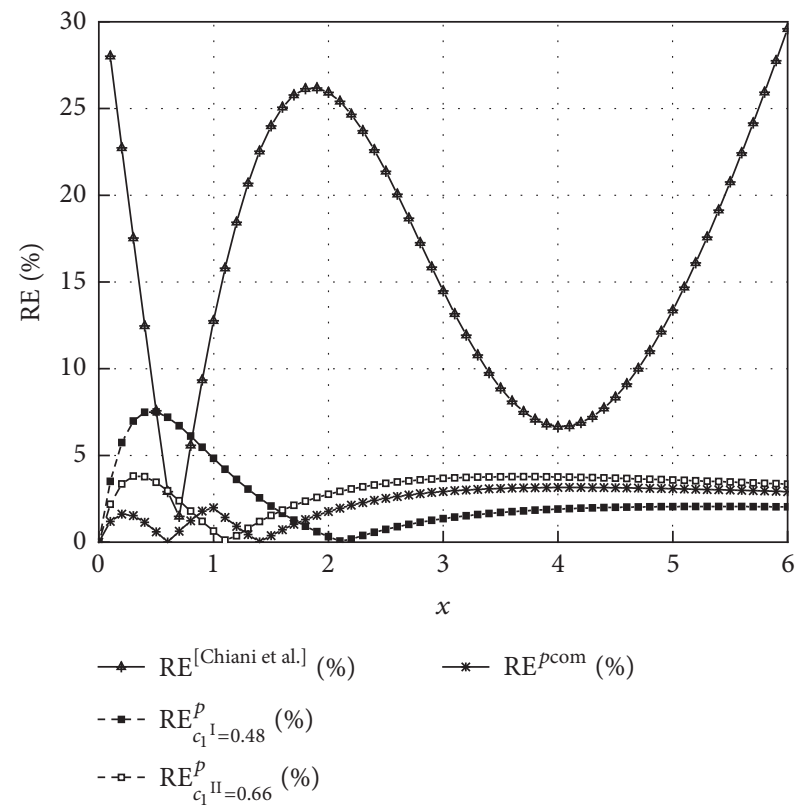

FIGURE 7: RE of the proposed approximation versus RE of the Chiani's et al. approximation.

Figures 4-10 provide the accuracy comparison of our approximation and the previously proposed approximations from the literature having comparable analytical form of approximation with respect to the one of the novel Qfunction approximation. It is worth noting from Figures 4-9 that, for $x \geq 2$, the accuracy of the approximation determined in accordance with the first criterion, $F^{p \mathrm{I}}(x)$, is comparable to or much better than that of the listed approximations from $[2-5,8]$. In contrast, the approximation obtained in 


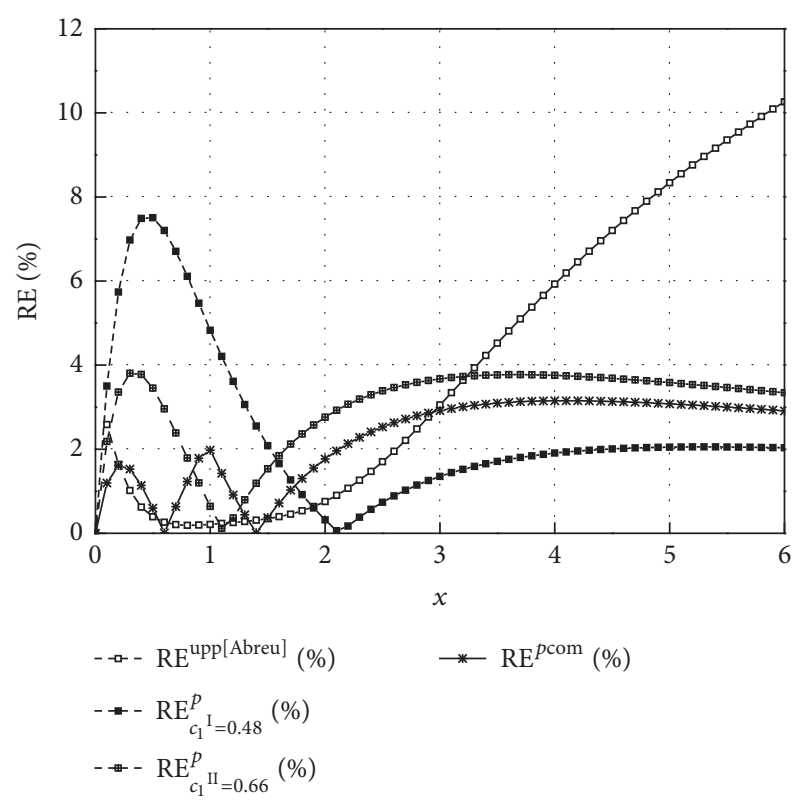

FIGURE 8: RE of the proposed approximation versus RE of the upper bound Abreu's approximation.

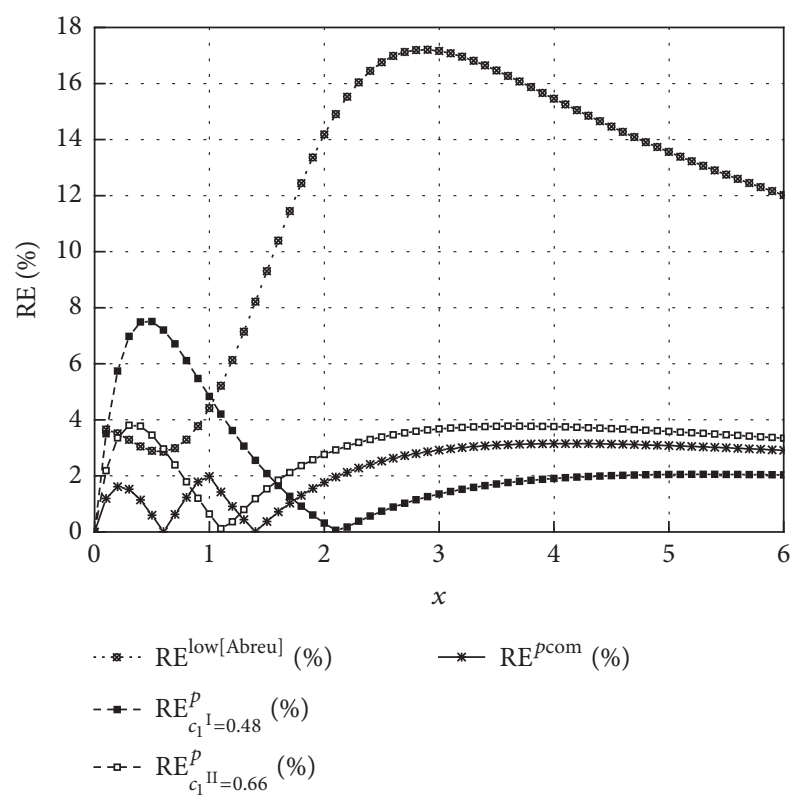

FIGURE 9: RE of the proposed approximation versus RE of the lower bound Abreu's approximation.

accordance with the second criterion, $F^{p \mathrm{II}}(x)$, is very accurate for $x<2$, that is, for small argument values. By comparing the complexity of the analytical forms of the approximations from $[2,3]$, with the one of the proposed approximation, we have already noticed that our approximation has lower analytical form complexity. As one can observe from Figure 4 the approximation proposed in [2] is better in terms of accuracy only for very small argument values. Another interesting observation that should be highlighted here is that, in the observed argument region, our approximation provides

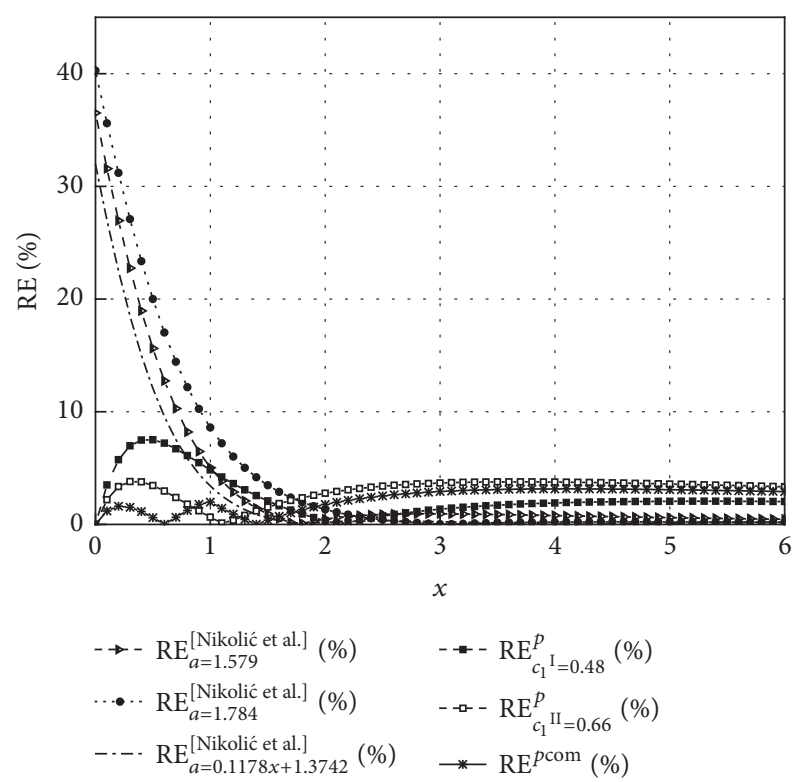

FIGURE 10: RE of the proposed approximation versus RE of the approximations from [19].

much better accuracy compared to Jang's approximation (see Figure 5). Moreover, by comparing the features of the proposed approximation and the ones of the approximation from [4], we can conclude that our approximation provides accuracy that is comparable to or even better than that of the approximation from [4] (see Figure 6), which comes at the cost of slightly increased analytical form complexity of our approximation compared to Fan's approximation. This complexity increase can be observed by comparing the analytical form of the denominators in (4) and (11). As we have already highlighted, the main advantage of Chiani's et al. approximation compared to the other approximations observed in this paper is its analytical simplicity. However, the main drawback of the Chiani's et al. approximation is a relatively small accuracy, which rather limits its application. One can notice from Figure 7 that in the observed argument region the proposed approximation determined for $c_{1}=c_{1}{ }^{\mathrm{II}}$ $=0.66$ completely outperforms Chiani's et al. approximation in terms of accuracy. Figure 8 shows that when $x$ is greater than 2 the approximation proposed by Abreu becomes less accurate. From Figure 9 one can notice that for the specified argument region our proposal given by (19) is more accurate than the one from [8].

Eventually, let us compare the accuracy of our novel Q-function approximation with the ones from [19]. From Figure 10 one can notice that the approximation we propose herein is much better in terms of accuracy compared to the one from [19], for the smaller values of arguments $(x \leq 2)$. This is due to the nice property of our approximation, already stated by (12). On the other side, for the higher arguments $(x>2)$, the approximations proposed in [19] are more accurate when compared to the novel one. Obviously, with the proper choice of the $c_{1}$ parameter value the observed difference in accuracy can be reduced [19]. 
Based on the obtained results we can derive as a final remark in this paper that further improvement of the presented results is likely to be achieved by combining our approximation with some of the previously reported ones. Specifically, by inspecting the curves presented in Figure 10 one can conclude that an additional improvement would also follow from the combination of our Q-function approximation and the one from [19], which has been left for the further studies. However, this improvement, as it is the one we have achieved with the composite method, will come at the cost of the increase of analytical form complexity. On the other side, low complexity of the analytical form of our originally proposed two-parametric approximation along with its relatively high accuracy makes her suitable for an accurate and efficient evaluation of many expressions with even intricate dependence on the Q-function. In brief, with the proposed approximation the accuracy is mostly better than that of the previously proposed approximations having similar or more complex analytical form. Eventually, we can conclude that, unlike some of the previously proposed approximations being very accurate for some argument regions and less accurate for the complement region, the approximation we have proposed in this paper has proved very accurate for all possible argument values, which stands for its advantage.

\section{Summary and Conclusions}

In this paper we have proposed the novel two-parametric approximation for the Q-function having relatively low analytical form complexity and providing relatively high accuracy comparable or even better than those of the previously proposed approximations having similar analytical form complexities. We have straightforwardly determined the one of the two parameters of our approximation, while we have examined the effect of the other parameter choice on the approximation accuracy. Moreover, we have specified two criterions for this parameter determining. By carefully examining the accuracy of the proposed approximation for a finite discrete small set of parameter values, as a corollary result, we have proposed one composite solution of the observed problem. With this solution, a notable improvement has been observed in terms of accuracy. We have anticipated that the simplicity of the proposed approximation enables its application over a wide range of theoretical studies. Moreover, we have highlighted that a relatively high accuracy of the proposed approximation observed for all argument values additionally extends its usability area to the applications that require high estimation accuracies. Eventually, we can conclude that the results presented in this paper can be practically used for many analyses involving the $Q$-function.

\section{Conflicts of Interest}

The authors declare that they have no conflicts of interest.

\section{References}

[1] C. Tellambura and A. Annamalai, "Efficient computation of erfc(x) for large arguments," IEEE Transactions on Communications, vol. 48, no. 4, pp. 529-532, 2000.
[2] G. K. Karagiannidis and A. S. Lioumpas, "An improved approximation for the Gaussian Q-function," IEEE Communications Letters, vol. 11, no. 8, pp. 644-646, 2007.

[3] W. M. Jang, "A simple upper bound of the gaussian q-function with closed-form error bound," IEEE Communications Letters, vol. 15, no. 2, pp. 157-159, 2011.

[4] P. Fan, "New inequalities of Mill's ratio and application to the inverse Q-function approximation," The Australian Journal of Mathematical Analysis and Applications, vol. 10, no. 1, pp. 1-11, 2013.

[5] M. Chiani, D. Dardari, and M. K. Simon, "New exponential bounds and approximations for the computation of error probability in fading channels," IEEE Transactions on Wireless Communications, vol. 2, no. 4, pp. 840-845, 2003.

[6] P. O. Borjesson and C.-E. W. Sundberg, "Simple Approximations of the Error Function $Q(x)$ for Communications Applications," IEEE Transactions on Communications, vol. 27, no. 3, pp. 639-643, 1979.

[7] A. Gasull and F. Utzet, "Approximating Mills ratio," Journal of Mathematical Analysis and Applications, vol. 420, no. 2, pp. 1832-1853, 2014.

[8] G. Abreu, "Very simple tight bounds on the Q-function," IEEE Transactions on Communications, vol. 60, no. 9, pp. 2415-2420, 2012.

[9] M. López-Benítez and F. Casadevall, "Versatile, accurate, and analytically tractable approximation for the Gaussian Qfunction," IEEE Transactions on Communications, vol. 59, no. 4, pp. 917-922, 2011.

[10] Y. Chen and N. C. Beaulieu, "A simple polynomial approximation to the Gaussian Q-function and its application," IEEE Communications Letters, vol. 13, no. 2, pp. 124-126, 2009.

[11] Y. Isukapalli and B. D. Rao, "An analytically tractable approximation for the Gaussian Q-function," IEEE Communications Letters, vol. 12, no. 9, pp. 669-671, 2008.

[12] Q. Shi and Y. Karasawa, "An accurate and efficient approximation to the gaussian Q-function and its applications in performance analysis in nakagami-m fading," IEEE Communications Letters, vol. 15, no. 5, pp. 479-481, 2011.

[13] V. N. Bao, L. P. Tuyen, and H. H. Tue, "A Survey on Approximations of One-Dimensional Gaussian Q-Function," REV Journal on Electronics and Communications, vol. 5, no. 1-2, 2015.

[14] Z.-H. Yang and Y.-M. Chu, "On approximating Mills ratio," Journal of Inequalities and Applications, vol. 273, 14 pages, 2015.

[15] M. Abramowitz and I. A. Stegun, Handbook of Mathematical Functions: with Formulas, Graphs, And Mathematical Tables, vol. 55, Courier Corporation, 1964.

[16] M. K. Simon, Probability Distributions Involving Gaussian Random Variables: A Handbook for Engineers And Scientists, Springer Science \& Business Media, 2007.

[17] I. Develi, "A new approximation based on the differential evolution algorithm for the Gaussian Q-function," International Journal of Innovative Computing, Information and Control, vol. 8, no. 10, article 70957102, 2012.

[18] J. Park and S. Park, "Approximation for the two-dimensional gaussian Q-function and its applications," ETRI Journal, vol. 32, no. 1, pp. 145-147, 2010.

[19] J. Nikolić, Z. Perić, and A. Jovanović, "Novel approximations for the Q-function with application in SQNR calculation," Digital Signal Processing, vol. 65, pp. 71-80, 2017.

[20] H. Vazquez-Leal, R. Castaneda-Sheissa, U. Filobello-Nino, A. Sarmiento-Reyes, and J. Sanchez Orea, "High accurate simple 
approximation of normal distribution integral," Mathematical Problems in Engineering, vol. 2012, Article ID 124029, 22 pages, 2012.

[21] Z. Perić and J. Nikolić, "Asymptotic analysis of switched uniform polar quantization for memoryless gaussian source," IEEE Signal Processing Letters, vol. 20, no. 1, pp. 75-78, 2013.

[22] S. Na, "Asymptotic formulas for variance-mismatched fixedrate scalar quantization of a Gaussian source," IEEE Transactions on Signal Processing, vol. 59, no. 5, pp. 2437-2441, 2011.

[23] A. Ž. Jovanović, Z. H. Perić, J. R. Nikolić, and M. R. Dinčić, "Asymptotic analysis and design of restricted uniform polar quantizer for Gaussian sources," Digital Signal Processing, vol. 49, pp. 24-32, 2016.

[24] N. S. Jayant and P. Noll, Digital Coding of Waveforms: Principles And Applications to Speech And Video, Prentice-Hall, Upper Saddle River, NJ, USA, 1984.

[25] P. Nazari, B.-K. Chun, F. Tzeng, and P. Heydari, "Polar quantizer for wireless receivers: Theory, analysis, and CMOS implementation," IEEE Transactions on Circuits and Systems I: Regular Papers, vol. 61, no. 3, pp. 877-887, 2014.

[26] Y. Hou, G. Liu, Q. Wang, and W. Xiang, "Performance optimization of digital spectrum analyzer with gaussian input signal," IEEE Signal Processing Letters, vol. 20, no. 1, pp. 31-34, 2013.

[27] Z. H. Peric, M. R. Dincic, and M. D. Petkovic, "The general design of asymptotic unrestricted polar quantizers with square cells," Digital Signal Processing, vol. 23, no. 5, pp. 1731-1737, 2013.

[28] Z. Perić and J. Nikolić, "Design of asymptotically optimal unrestricted polar quantizer for gaussian source," IEEE Signal Processing Letters, vol. 20, no. 10, pp. 980-983, 2013.

[29] J. Nikolić, Z. Perić, and A. Jovanović, “Two forward adaptive dual-mode companding scalar quantizers for Gaussian source," Signal Processing, vol. 120, pp. 129-140, 2016.

[30] Z. Perić, J. Lukić, J. Nikolić, and D. Denić, “Application of meansquare approximation for piecewise linear optimal compander design for gaussian source and gaussian mixture model," Information Technology and Control, vol. 42, no. 3, pp. 277-285, 2013.

[31] J. Nikolić and Z. Perić, "Optimal compressor function approximation utilizing Q-function approximations," Facta Universitatis, Series: Automatic Control and Robotics, vol. 15, no. 2, pp. 85-94, 2016.

[32] D. Lu, "Certain approximations to achieve sharp lower and upper bounds for the Mills' ratio of the inverse Gaussian distribution," Journal of Mathematical Analysis and Applications, vol. 444, no. 1, pp. 737-744, 2016.

[33] E. Camponogara and L. F. Nazari, "Models and algorithms for optimal piecewise-linear function approximation," Mathematical Problems in Engineering, vol. 2015, Article ID 876862, 9 pages, 2015.

[34] S. M. Stefanov, "On the application of iterative methods of nondifferentiable optimization to some problems of approximation theory," Mathematical Problems in Engineering, vol. 2014, Article ID 165701, 10 pages, 2014. 


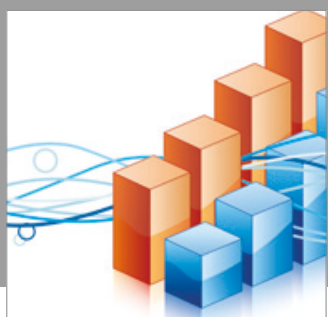

Advances in

Operations Research

vatersals

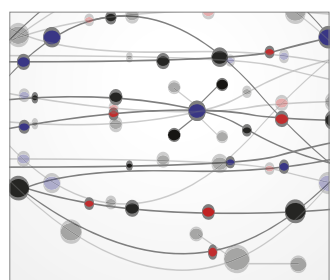

\section{The Scientific} World Journal
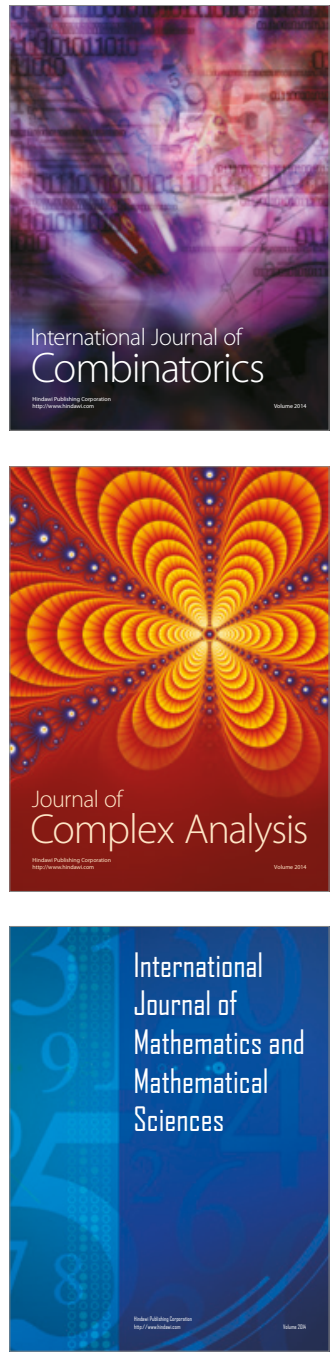
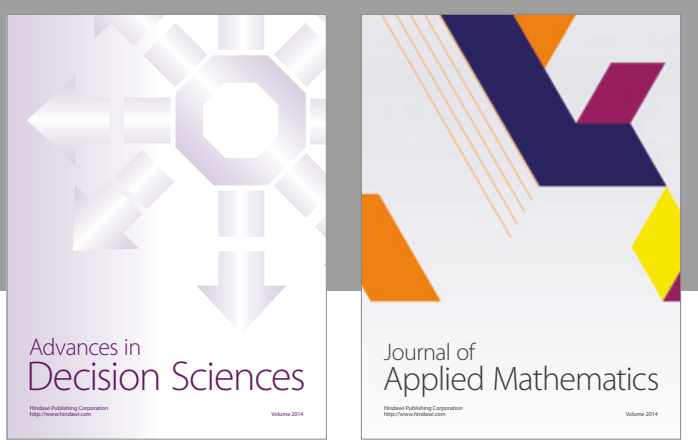

Algebra

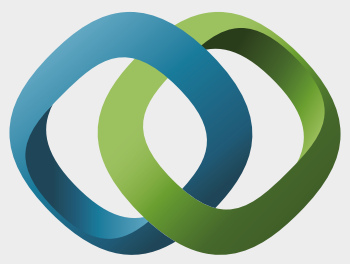

\section{Hindawi}

Submit your manuscripts at

https://www.hindawi.com
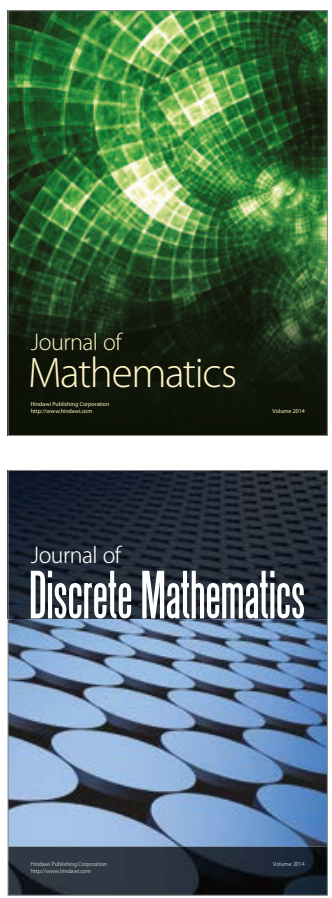

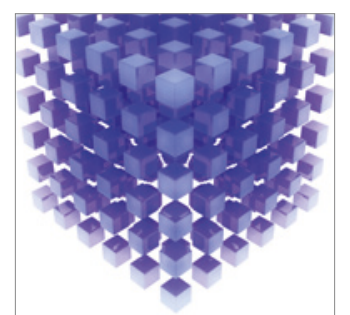

Mathematical Problems in Engineering
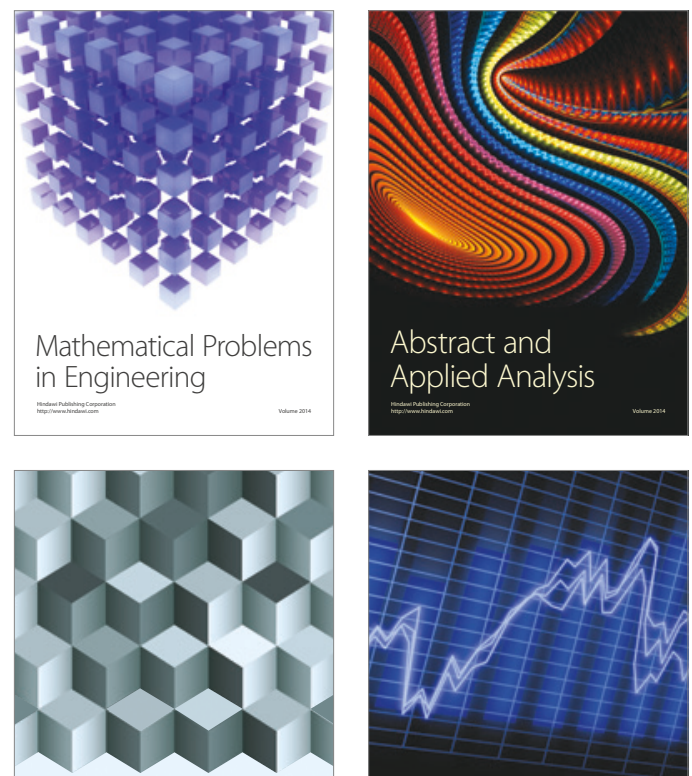

Journal of

Function Spaces

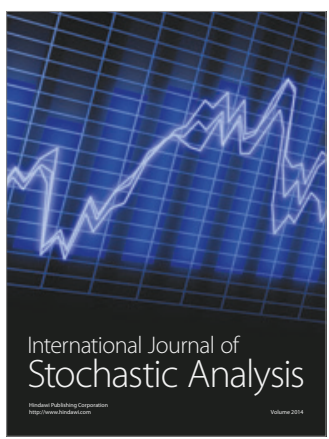

Probability and Statistics
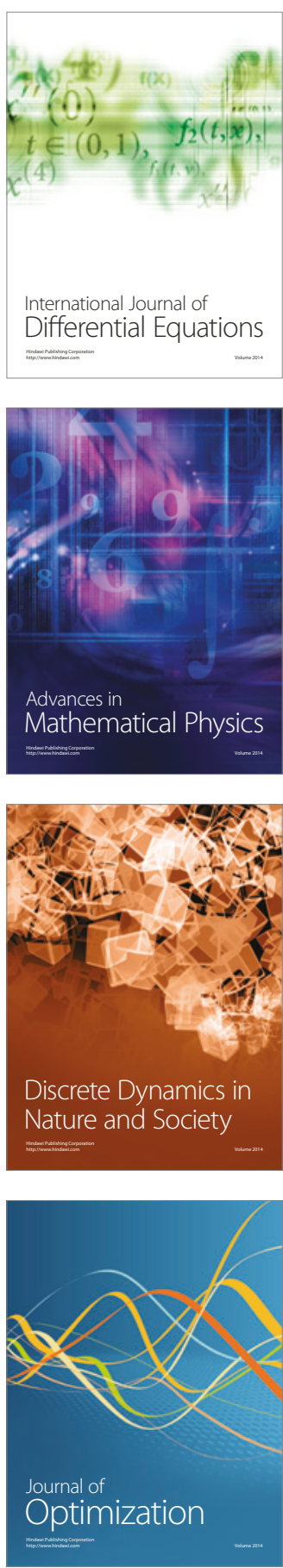